\title{
14th international symposium on high pressure low temperature plasma chemistry (HAKONE XIV)
}

The 14th international symposium on high pressure low temperature plasma chemistry (HAKONE XIV) was held in Zinnowitz on the second largest german island Usedom at the Baltic Sea from September 21st to 26th 2014. The conference was jointly organized by the Institute of Physics at the University of Greifswald and the Leibniz Institute for Plasma Science and Technology (INP Greifswald). With HAKONE XIV a series of successful conferences devoted to the fundamentals and applications of non-thermal plasmas at elevated pressures was continued. 154 regular attendees and 15 accompanying persons and guests from 21 different countries participated and submitted 139 contributions in 9 different topics.

HAKONE is a bi-annual symposium bringing together scientists and engineers working on subjects in the basic research and technology of high pressure (typically 1 bar) and low temperature plasma chemistry. Besides the traditional topics dealing with fundamental aspects of gas discharges at elevated pressures the fields of surface processing and technology, environmental applications and biological applications were strongly represented. Five invited lectures covered the following topics:

- Physical mechanisms of self-organization and formation of current patterns in gas discharges by Mikhail Mokrov and Yuri P. Raizer, Russia.

- Spectroscopic diagnostics of atmospheric pressure and underwater corona discharges by Kunihide Tachibana, Japan.

- Diagnostics of non-equilibrium plasmas and their interaction with liquids for biomedical applications by Peter J. Bruggeman, USA.

- Dielectric barrier discharge based polymer surface nitrogenation by Claus-Peter Klages, Germany.

- Application and Mechanism of Plasma-catalysis by Christopher Whitehead, UK.

For the first time at HAKONE a best poster award was presented to students/PhD-students at the conference, sponsored by Wiley VCH. The members of the International Scientific and Organizing Committee (ISOC) elected Marc Bogaczyk and Robert Tschiersch (University of Greifswald) with their contribution on the development of surface charges on the dielectrics of barrier discharges. The support of Wiley VCH and the other supporters of HAKONE XIV, namely the collaborative research center "Fundamentals of complex plasmas" (TRR 24), Deutsche Forschungsgemeinschaft DFG, European Union (in the scope of project "PlasmaShape"), Wirtschaftsfördergesellschaft Vorpommern and Invest-in-MV is gratefully acknowledged.

Dr. Ulrich Kogelschatz and Prof. Chobei Yamabe retired from the ISOC which is now chaired by Prof. Mirko Cernak (Masaryk University Brno). The ISOC and the Local Organizing Committee of HAKONE XIV are grateful to the two retired colleagues for their outstanding contribution and unfatiguing support of the symposium for more than 20 years.

The IOSC continued the collaboration with the European Physical Journal - Applied Physics and prepared this special issue of the journal devoted to the conference. It contains one summary paper based on the invited talk given by K. Tachibana and 10 papers based on original presentations. All contributed papers of the HAKONE XIV symposium are available in the conference proceedings distributed electronically to all participants. These documents and all other information are also available at the conference web-site www.hakone2014.org.

Finally we would like to thank all participants of HAKONE XIV and invite the readers of the journal to the next HAKONE symposium, which will be held in fall 2016 in Brno, Czech Republic.

Ronny Brandenburg and Lars Stollenwerk

Chairpersons of the Local Organizing Committee

Mirko Černak

Chairman of the International Scientific and Organizing Committee 